\title{
Exite2 Observation of the Sigma Source Grs $1227+025$
}

\section{Citation}

Grindlay, J. E., Y. Chou, P. F. Bloser, and T. Narita. 2005. "EXITE2 Observation of the SIGMA Source GRS 1227+025." The Astrophysical Journal 618 (2): 852-55. https:// doi.org/10.1086/383457.

\section{Permanent link}

http://nrs.harvard.edu/urn-3:HUL.InstRepos:41399920

\section{Terms of Use}

This article was downloaded from Harvard University's DASH repository, and is made available under the terms and conditions applicable to Other Posted Material, as set forth at http:// nrs.harvard.edu/urn-3:HUL.InstRepos:dash.current.terms-of-use\#LAA

\section{Share Your Story}

The Harvard community has made this article openly available.

Please share how this access benefits you. Submit a story.

Accessibility 


\title{
EXITE2 Observation of the SIGMA Source GRS 1227+025
}

\author{
J.E. Grindlay \\ Harvard-Smithsonian Center for Astrophysics, Cambridge, MA 02138 \\ Y. Chou \\ National Tsing Hua University, Hsinchu, Taiwan, Republic of China \\ P.F. Bloser \\ NASA/Goddard Space Flight Center, Greenbelt, MD 20771 \\ and \\ T. Narita \\ College of the Holy Cross, Worcester, MA 01610 \\ jgrindlay@cfa.harvard.edu
}

\begin{abstract}
We report the EXITE2 hard X-ray imaging of the sky around 3C273. A $2 \mathrm{~h}$ observation on May 8, 1997, shows a $\sim 260$ mCrab source detected at $\sim 4 \sigma$ in each of two bands (50-70 and 70-93 keV) and located $\sim 30$ ' from 3C273 and consistent in position with the SIGMA source GRS1227+025. The EXITE2 spectrum is consistent with a power law with photon index 3 and large low energy absorption, as indicated by the GRANAT/SIGMA results. No source was detected in more sensitive followup EXITE2 observations in 2000 and 2001 with $3 \sigma$ upper limits of 190 and 65 mCrab, respectively. Comparison with the flux detected by SIGMA shows the source to be highly variable, suggesting it may be non-thermal and beamed and thus the first example of a "type 2" (absorbed) Blazar. Alternatively it might be (an unprecedented) very highly absorbed binary system undergoing accretion disk instability outbursts, possibly either a magnetic CV, or a black hole X-ray nova.
\end{abstract}




\section{Introduction}

GRS1227+025 was discovered serendipitously with SIGMA (Bassani et al. 1991; Jourdain et al. 1992) in the 40-80 keV energy band during an observation of the QSO 3C273. Separated from 3C273 by only 15', GRS1227+025 (hereafter GRS1227) was detected at $5.5 \sigma$ whereas $3 \mathrm{C} 273$ was not detected. The source has a significantly steeper spectrum (photon index $3_{-0.9}^{+1.3}$, Bassani et al. (1991)) compared to 3C273, which was detected in subsequent observations, with its usual flatter photon index of $\sim 1.5$. Upper limits (15-30 keV) with ART-P (simultaneous with SIGMA) showed that the spectrum must be heavily absorbed: $\mathrm{N}_{H} \sim 1.5 \times 10^{25} \mathrm{~cm}^{-2}$ for a power law spectrum with photon index 3 (Bassani et al. 1993). Followup observations done with SIGMA and OSSE (which could only measure the combined flux of 3C273 and GRS1227) showed that GRS1227 is probably variable by a factor 2-5 on $\sim 1$ day time scales (Bassani et al. 1993; Johnson et al. 1995). No obvious soft X-ray counterpart was found in the SIGMA error circle (5 arcmin, $1 \sigma)$ with ROSAT (e.g. Leach \& McHardy (1996)), although an Einstein source (1E 1227+0224) in the SIGMA error circle $(1 \sigma)$ was identified with a QSO at $\mathrm{z}=0.57$ that would have to be of "type 2" (heavily self-absorbed) if it were indeed the counterpart (Grindlay 1993).

\section{EXITE2 Observation and Data Analysis}

The second-generation Energetic X-ray Imaging Telescope Experiment (EXITE2) was a phoswich $(\mathrm{NaI}(\mathrm{Tl}) / \mathrm{CsI}(\mathrm{Na}))$ imaging telescope designed to detect and image cosmic X-ray sources in the broad hard X-ray energy band $(20-600 \mathrm{keV})$ from a high-altitude balloon. The details of the payload are described in Lum et al (1994); Manandhar (1995); Chou et al. (1998); Chou (2001); Chou et al. (2003). EXITE2 flew three times (from Ft. Sumner, New Mexico) in 1997, 2000 and 2001 for scientific observations, which included the Crab Nebula, the X-ray binaries Cyg X-1 and 4U 0614+09, the microquasar GRS 1915+105, the Seyfert galaxy NGC 4151 and QSO 3C273/GRS1227. The data analysis methods and the observation results of the Crab and Cyg X-1 are reported in separate papers (Bloser et al. 2002a; Chou et al. 2003). Other results from the 2000 and 2001 flights, including separate experiments for the development of imaging Cd-Zn-Te detectors (Bloser et al 2002b, Jenkins et al 2003) are reported elsewhere.

For the 1997 flight, during UT03:20 to UT05:32 on May 8 (local time 21:20 to 23:32 on May 7), the telescope was pointing to within 5 arcmin of the QSO 3C273 at (night) flight altitude $\sim 115,000$ feet. The data were analyzed by the EXITE2 standard analysis methods described in Chou (2001), Bloser et al. (2002a) and Chou et al. (2003). The sky images showed that no significant $(>3 \sigma)$ detection of $3 \mathrm{C} 273$ was obtained during the $\sim 2$ hour 
observation. However, a source $~ 30$ ' away from 3C273 was detected in the EXITE2 energy band $5(50-70 \mathrm{keV})$ and band $6(70-93 \mathrm{keV})$ at $3.9 \sigma$ and $4.1 \sigma$, respectively. We estimated aspect errors as $\lesssim 5$ ' from agreement of the Crab and Cyg X-1 images with their source positions. The image for band 6 is shown as Figure 1.

The source detected by EXITE2 in 1997 near 3 C273 was located at $\alpha(2000)=12^{h} 31^{m} 13^{s}$, $\delta(2000)=+2^{\circ} 18$ ', with positional uncertainty $\pm \sim 7$ arcmin $(1 \sigma)$. Although $\sim 18^{\prime}$ away from the GRS1227 location reported by Jourdain et al (1992) and plotted in Figure 2, the $2 \sigma$ error circles for SIGMA vs. EXITE2 overlap. The detected energy bands and the combined $5 \sigma$ significance of the EXITE2 detection are both comparable to the original SIGMA detection, further supporting the reality of a highly variable source at this location and distinct from $3 \mathrm{C} 273$. The fluxes measured during the 2 hours observation were $(6.6 \pm 1.7) \times 10^{-4}$ photons $\mathrm{cm}^{-2} \mathrm{~s}^{-1} \mathrm{keV}^{-1}$ and $(3.5 \pm 0.85) \times 10^{-4}$ photons $\mathrm{cm}^{-2} \mathrm{~s}^{-1} \mathrm{keV}^{-1}$, for band 5 and 6 respectively, a factor of $\sim 10$ brighter than SIGMA 1990 observations. The combined band 5-6 flux was $(4.94 \pm 0.91) \times 10^{-4}$ photons $\mathrm{cm}^{-2} \mathrm{~s}^{-1} \mathrm{keV}^{-1}(\sim 260 \mathrm{mCrab})$. No source was significantly detected in band $4(37-50 \mathrm{keV})$ or band $7(93-127 \mathrm{keV})$ with $3 \sigma$ upper limits $1.8 \times 10^{-3}$ photons $\mathrm{cm}^{-2} \mathrm{~s}^{-1} \mathrm{keV}^{-1}$ and $1.2 \times 10^{-4}$ respectively. We found that a spectrum of power law index $\sim 3$ as reported by Bassani et al. 1991 for GRS1227 is consistent with the EXITE2 1997 observation results (see Figure 3). Alternatively, the source could still have a flatter spectrum (e.g. photon index $\sim 1.7$ as for typical AGN) with high energy cutoff at $\sim 100 \mathrm{keV}$.

Additional observations of the field containing 3C273/GRS1227 were made in the EXITE2 flights on September 192000 (UT20:45 to UT21:30) and May 232001 (UT03:09 to UT06:17 on May 24). No source was detected during the $\sim 45$ minute observation in the 2000 flight. The $3 \sigma$ upper limit for the combined bands 5-6 (50 to $93 \mathrm{keV}$ ) was $3.6 \times 10^{-4}$ photons $\mathrm{cm}^{-2} \mathrm{~s}^{-1} \mathrm{keV}^{-1}(\sim 190 \mathrm{mCrab})$, which is a factor 1.5 below the 1997 value. For the 2001 observation, the telescope was pointed to the combined position of SIGMA/EXITE2, the intersection of the $2 \sigma$ error circles for EXITE2 and SIGMA at RA $=12^{h} 30^{m} 14^{s}$, DEC $=$ $+2^{\circ} 14 ' 53$ " (J2000), as shown in Figure 2. No source was detected during a $\sim 3$ hour observation. The $3 \sigma$ upper limit for the combined bands 5-6 (50 to $93 \mathrm{keV}$ ) was $1.25 \times 10^{-4}$ photons $\mathrm{cm}^{-2} \mathrm{~s}^{-1} \mathrm{keV}^{-1}$, or $\sim 65 \mathrm{mCrab}$, which is comparable to the original SIGMA detection. The upper limits for both EXITE2 observations are also plotted in Figure 3. The combined EXITE2-SIGMA observations indicate that the source is highly variable (by a factor of $\sim 10$ ) at hard X-ray energies. 


\section{Discussion}

The GRS1227 source presents a major puzzle. Both EXITE2 and SIGMA detect a source at approximately the same location (within $2 \sigma$ ) and with similar spectral properties which are very distinct from 3C273. Although the EXITE2 detection is marginal, as was the original SIGMA detection, the combined results are more likely to be real. GRS1227 must be highly self-absorbed and yet highly variable. The SIGMA variations are a factor of $\sim 2$ and SIGMA-EXITE2 variation is nearly a factor of $\sim 20$. This is a combination not seen before, and suggests a new class of object: a type 2 (i.e. heavily absorbed) Blazar, since Blazars (BL Lacs) are usually the only AGN which show such extreme variability. However, it might also be a Narrow Line Seyfert 1 (NLSy) galaxy (e.g. RX J2217.9-5941, see Grupe et al. (2001)) but must still be highly self-absorbed. It may be similar to the NLSys with significant internal absorption and significant variability described by Fabian \& Iwasawa (1999). Alternatively it might be (an unprecedented) very highly absorbed binary system, possibly a low luminosity magnetic CV undergoing outburst, since intermediate polars at high inclination can also show significant internal absorption.

From the EXITE2 vs. SIGMA source error circles $(2 \sigma)$ shown in Figure 2, the combined source error box is $\sim 15^{\prime} \times 6^{\prime}$, centered on the EXITE2-SIGMA combined position $\mathrm{RA}=12^{h} 30^{m} 14^{s}\left( \pm 4^{\prime}\right), \mathrm{DEC}=+2^{\circ} 14^{\prime} 53^{\prime \prime}\left( \pm 4^{\prime}\right)$ (J2000). At this relatively uncrowded high latitude position, even a $\sim 2$ ' position could enable identification with an optical or radio variable. However the need for a $\lesssim 1$ ' position is obvious: if this is, as suspected, a highly absorbed non-thermal source (BL Lac), it may not be at all conspicuous in the optical and appear as a simple elliptical galaxy. The radio galaxies numbered (2) and (4) in Figure 2, or [SRK80]122803+023451 and 87GB[BWE91]1227+0230, are thus possible candidates. However, in the event that the radio emission is confined to the core, GRS1227 may not even be bright at radio wavelengths since it is likely Compton-thick. Given the space density of galaxies cataloged (SIMBAD and NED) within the combined EXITE2/SIGMA error circles (cf. Figure 2), a $\lesssim 1$ ' source position is obviously needed for a unique galaxy identification.

Source variability measurements can partly constrain the underlying source nature. The generalized condition for maximum variability of an isotropically emitting source powered by accretion at rate $\dot{m}$ and radiating luminosity $\mathrm{L}_{x}$ is

$$
\Delta \mathrm{L}_{x} \lesssim 2 \times 10^{41} \eta_{0.1} \Delta \mathrm{t} \mathrm{ergs} / \mathrm{s}
$$

where $\eta_{0.1}=\mathrm{L}_{x} / \dot{m} c^{2} \sim 0.1$ is the likely maximum efficiency factor (cf. Guilbert et al. (1983)). This can impose direct constraints on the source luminosity (and thus distance and 
nature) for an observed luminosity variation $\Delta \mathrm{L}_{x}$ over time interval $\Delta \mathrm{t}$. Therefore, a factor of 2 increase on $\sim 1 \mathrm{~d}$ timescales (already suspected from SIGMA) would imply the source is beamed and thus a Type 2 Blazar if it were identified with an AGN at z $\gtrsim 0.1$ (like 3C273) with $\mathrm{L}_{x} \gtrsim 10^{45} \mathrm{erg} / \mathrm{s}$.

GRS1227 exhibits an unusual spectral shape. The simultaneous detection by SIGMA ( $240 \mathrm{keV}$ ) and upper limit at $\lesssim 15 \mathrm{keV}$ by the ART-P X-ray imager on GRANAT (Bassani 1991 ) indicated that the source is highly self-absorbed $\left(\mathrm{N}_{H} \sim 10^{24-25} \mathrm{~cm}^{-2}\right)$ and thus a Compton-thick absorber (see Matt et al. (1999) and references therein). If the spectrum above $\sim 40-50 \mathrm{keV}$ is indeed a power law with photon index $\sim 3$, it is consistent with a Blazar or NLSy. Although NLSys are also highly variable, these have not been reported as Type 2 objects. As mentioned above, the EXITE2 spectral shape (Figure 3) is equally consistent with a flatter spectrum with photon index 1.7 but high energy cutoff at $\sim 100 \mathrm{keV}$, as expected for a highly absorbed Seyfert 2 or Type 2 QSO. However the remarkable variability required argues against the Seyfert or Type 2 QSO interpretation and in favor of a Blazar, with highly variable non-thermal emission. In this case, large amplitude optical variability might also be expected, since Liller \& Liller (1975) found a 5 magnitude brightening for the BL Lac PKS 1510-089 on the Harvard plates. Given the offset from 3C273, it is possible that significant optical variables could have been missed, and a search using the Harvard plates is in progress.

A magnetic CV interpretation is also possible even if the spectrum is indeed a (moderately steep) power law: the magnetic CV AE Aqr can be fit with a similar power law spectrum (Eracleous \& Horne 1996), and has been reported as a high energy gamma-ray source (cf. Meintjes and de Jager 2000 and references therein). A magnetic CV origin for GRS1227 might also explain the extreme flare variations: the object could be a dwarf nova (like GK Per) undergoing outbursts. Although the X-ray emission in classical dwarf novae outbursts is usually soft, an outburst in a magnetic CV may contain significant non-thermal emission. Historical optical measurements of the could again provide an important test, since the optical flux (from the disk) would be expected to increase by $\sim 5$ mag. If the EXITE2 1997 detection was the peak of an outburst like that of GK Per, the expected X-ray optical flux ratio $\mathrm{F}_{x} / \mathrm{F}_{v} \sim 10$ would imply an outburst magnitude $\mathrm{V} \sim 10$, and thus quiescence magnitude $\mathrm{V} \sim 15$-16, which is probably too bright to have been missed as a blue object in the Palomar Green survey (Green, Schmidt and Liebert 1986), which revealed no such objects near 3C273.

A high latitude black hole X-ray binary, an X-ray nova (XN) like XTE J1118+480. which showed evidence for magnetic flares and non-thermal optical emission (Merloni, DiMatteo and Fabian 2000) is conceivable. Here the power law hard X-ray spectrum is natural; this is 
seen in the very high state of BH novae (e.g. Nova Muscae, Esin et al. (1999)) and is expected for the jet emission probable in a system like XTE J1118+480. It too would have to be highly self-absorbed, but - as with the CV hypothesis - is plausible if the system were at inclination $\sim 90^{\circ}$, and self-absorbed at the required column density of $\mathrm{N}_{H} \sim 10^{25} \mathrm{~cm}^{-2}$. Such a highly absorbed XN would be missed by the ASM on RXTE, and indeed no evidence for an ASM source is found at the time of the 1997 EXITE2 observation (or the 2000 and 2001 EXITE2 observations; cf. Figure 4). However, an optical outburst is again expected and should again be searched on historical monitoring (e.g. plate) data. Thus the galactic binary/transient scenarios might be distinguished from the Type 2 BLazar or NLSeyfert interpretation by historical optical or radio variations, which are probably larger in the binary case given the relatively low luminosity secondary star expected. The lack of any (large amplitude) optical variable would support the Type 2 Blazar or NLSeyfert interpretation.

Definitive confirmation of the source existence, and of course a refined hard X-ray position and spectrum, are obviously needed. Long after this paper was initially submitted, we have carried out an observation with INTEGRAL (IBIS telescope) to attempt to further measure the source position, spectrum and variability. Data have just been received and will be reported separately. An initial INTEGRAL/IBIS observation of 3C273 has been reported recently by Courvoisier et al (2003) which also provided a sensitive new upper limit $(\sim 10$ mCrab) for emission from the position of GRS1227 - a factor of $\sim 6$ below the SIGMA detection and 2001 EXITE2 upper limit and 25 below the 1997 EXITE2 detection. Thus the source variability at $\gtrsim 40 \mathrm{keV}$ must be at least this factor. Wide-field XMM observations of the 3C273 field should also be examined for evidence for a highly variable, highly absorbed $(\lesssim 10 \mathrm{keV})$ X-ray source which would enable identification.

The authors thank T Gauron, J. Gomes, V. Kuosmanen, F. Licata, G. Nystrom, A. Roy, R. Scovel (SAO) and J. Apple (MSFC) for for the EXITE2 detector and gondola development, and NSBF personnel for their excellent support of the balloon launches and flight operations. This work was partially supported by NASA grants NAGW-5103 and NAG5-5279. PFB is a National Research Council Research Associate at NASA/Goddard Space Flight Center. TN acknowledges support from the College of the Holy Cross.

\section{REFERENCES}

Bassani L. et al. 1999, Proc. of 22nd Int. Cosmic Ray Conf., 1, 173

Bassani L. et al. 1993, A\&A. 97, 89 
Bloser, P. F., Chou, Y., Grindlay, J. E., Narita, T., \& Monnelly, G., 2002a, Astroparticle Physics, 17, 393

Bloser, P., Narita, T., Jenkins, J., Perrin, M., Murray, R. and Grindlay, J. 2002b, Proc. SPIE, 4497, 88

Chou, Y., et al. 1998, in Conf. Record of the 1998 IEEE Nucl. Sci. Symposium (Piscataway, New Jersey: IEEE), 210

Chou, Y., 2001, Ph.D thesis, Harvard University

Chou, Y., Bloser, P. F., Grindlay, J. E., Jenkins, J. A., \& Narita, T., 2003, submitted to ApJ

Courvoisier, T., Beckmann, V., Bourban, G., Chenevez, J., Chernyakova, M., Deluit, S., Favre, P., Grindlay, J. et al. 2003, A\&A, in press (astro-ph/0308212)

Eracleous, M. and Horne, K. 1996, ApJ, 471,427

Esin, A. A., McClintock, J. E., \& Narayan, R., 1997, ApJ, 486, 865

Fabian, A. C. and Iwasawa, K., 1999, MNRAS, 303, L34

Green, R. F., Schmidt, M., and Liebert, J., 1986, Ap.J. Suppl., 61, 305

Grindlay, J. E. 1993, A\&AS, 97, 113

Grupe, D., Thomas, H. -C. and Leighly, K. M., 2001, A\&A, 369, 450

Guilbert, P., Fabian, A., \& Rees, M. 1983, MNRAS, 205, 593

Johnson, W. N. et al. 1995, ApJ, 445, 182

Jourdain, E. et al. 1992, ApJ, 395, L69

Leach, C. M. \& McHardy I. M., 1996, MNRAS, 278, 465

Liller , M. H. and Liller, W., 1975, ApJ, 199, L133

Lum, K. S. K. et al. 1994, IEEE Trans. on Nucl. Sci., NS-41, 1354

Manandhar, R. P. 1995, Ph.D. Dissertation, Harvard University

Matt, G., Pompilio, F. \& La Franca, F., 1999 New Astro. 4, 91

Meintjes, P.J. and de Jager, O.C. 2000, MNRAS, 311, 611 
Merloni, A., DiMatteo, T. and Fabian, A. 2000, MNRAS, 318, L15

This preprint was prepared with the AAS IATEX macros v5.2. 


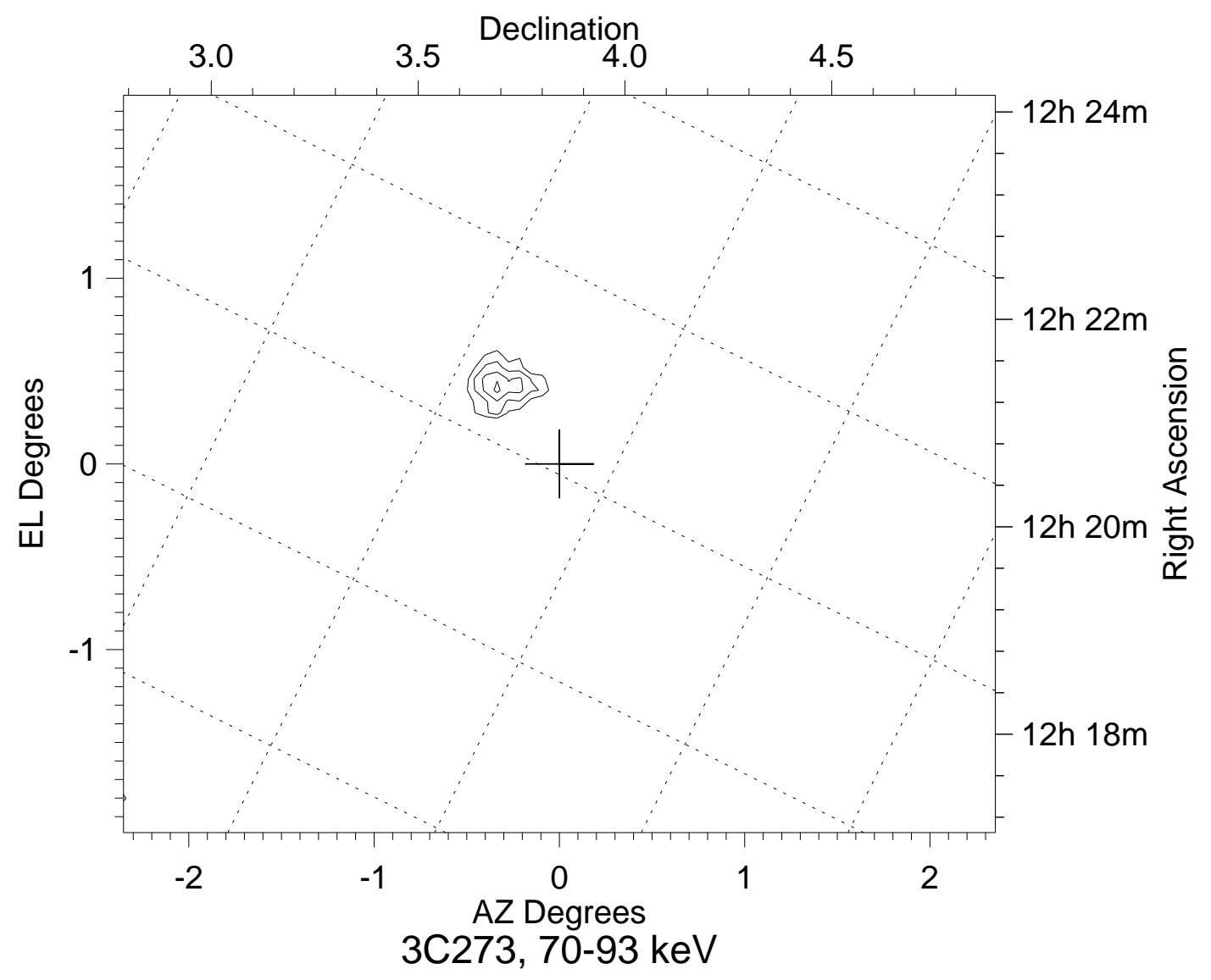

Fig. 1.- Image of GRS1227+025 obtained with EXITE2 on UTMay 8.17, 1997, in the 70 $93 \mathrm{keV}$ band. Contours begin at $2.5 \sigma$ and increase in steps of $0.5 \sigma$ to $\sim 4 \sigma$. The source is independently detected at $\sim 4 \sigma$ in the $50-73 \mathrm{keV}$ band. The expected position of $3 \mathrm{C} 273$ is shown by the cross; the source detected is at a position (J2000) compatible with the SIGMA detection of GRS1227+025. 


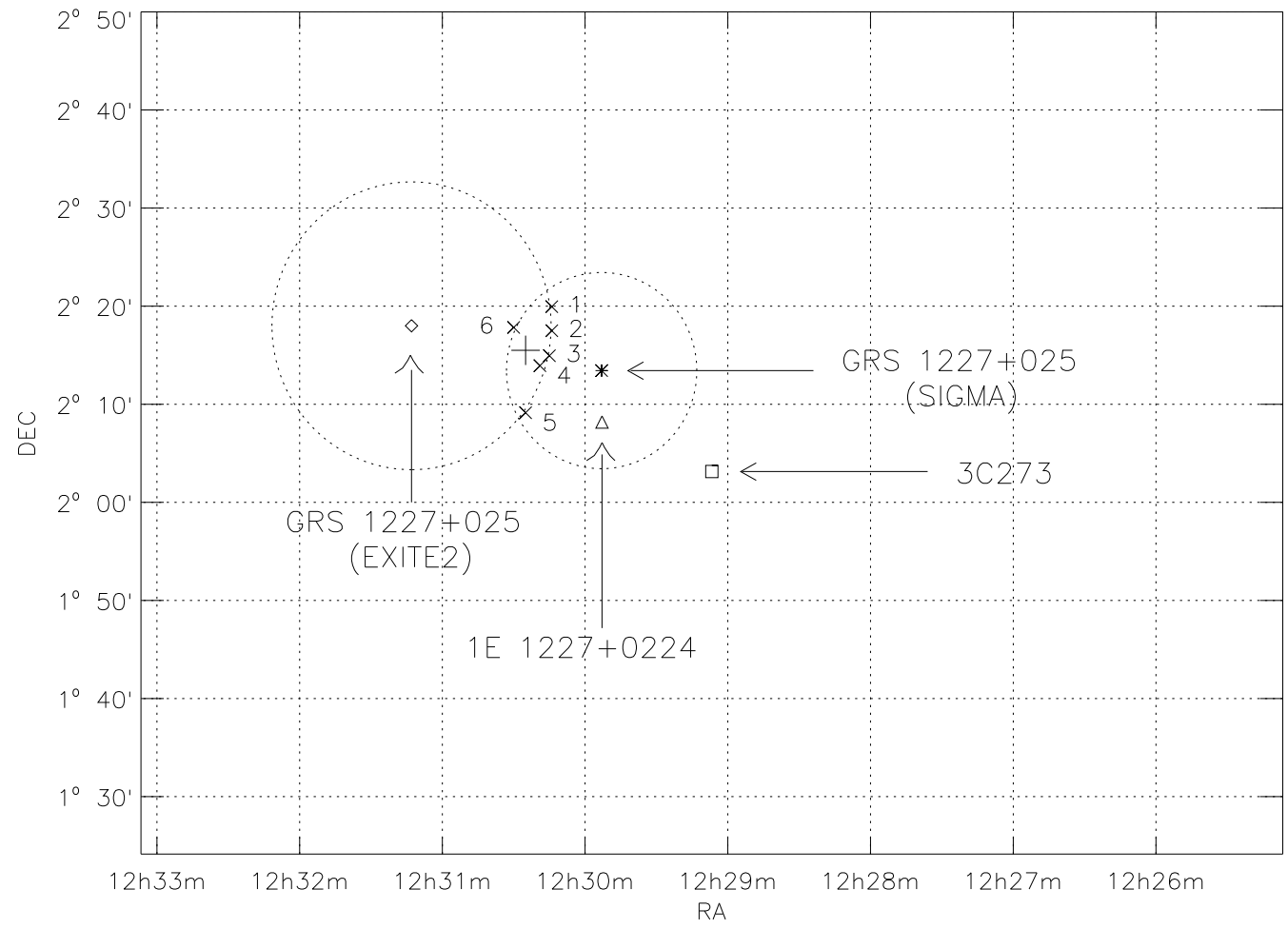

Fig. 2.- GRS1227+025 source positions (plotted on J2000 grid) measured ( $\sigma \sigma$ radius error circles) by SIGMA ( 10') and by EXITE2 ( 14'). The positions of 3C273 and 1E 1227+0224 are also shown. The cross marked inside the intersection of the $2 \sigma$ error circles is combined position of EXITE2 and SIGMA. The numbered source positions $(\mathrm{X})$ are galaxies $(1,3,5,6)$ and radio galaxies $(2,4)$ found in a SIMBAD and NED search of the intersecting error circle for EXITE2/SIGMA. 


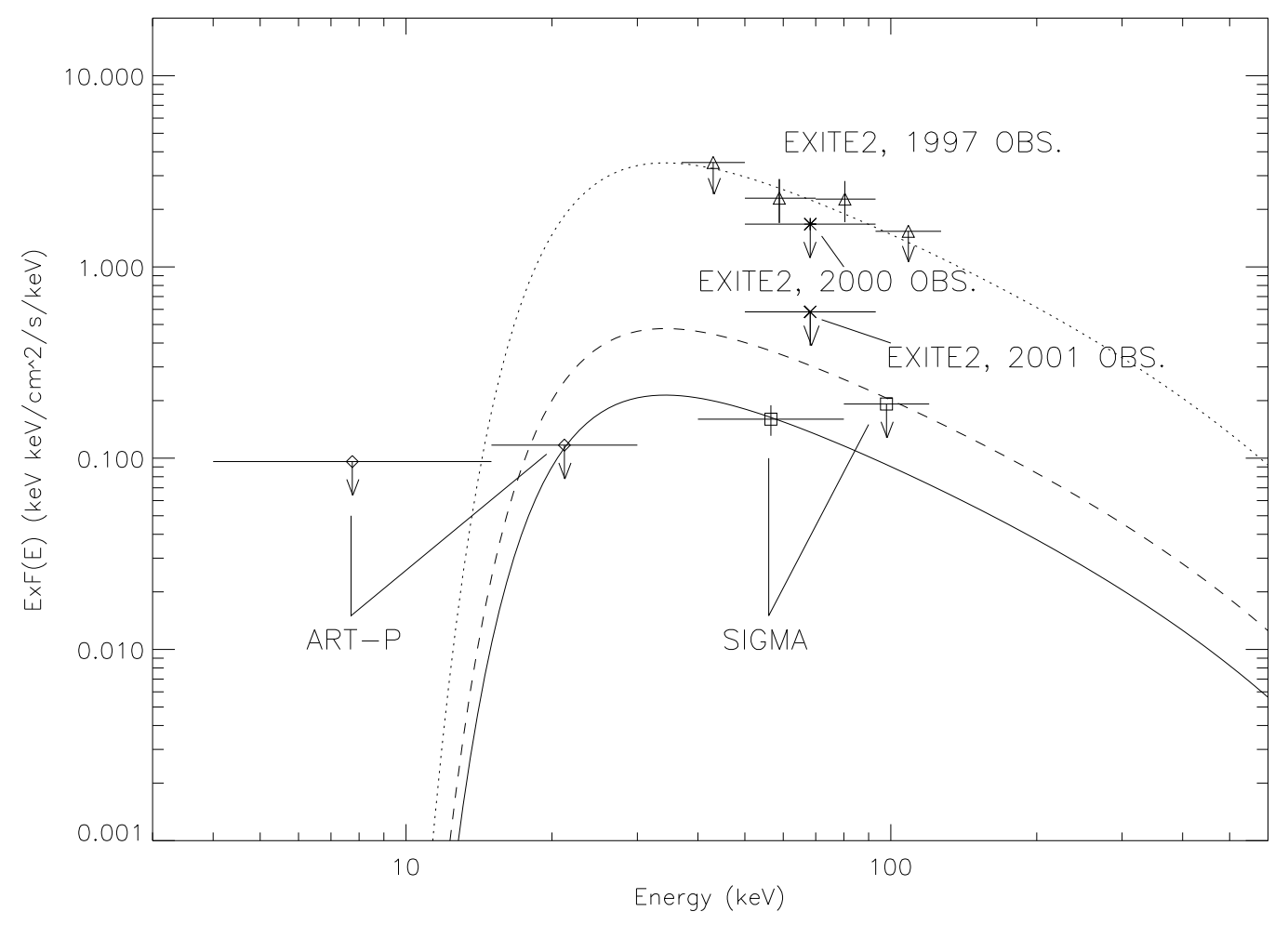

Fig. 3.- Spectrum measured by the 1997 EXITE2 observation (triangle data points and dotted curve), and the upper limit from the 2000 observation (asterisk data point) and 2001 observation ("X" data point) vs. that measured in the SIGMA/ART-P observations (square and diamond points; from Jourdain et al. (1992) and dashed curve, from Bassani et al. (1991)). Each of the three curves is for a power law with photon index 3 and hydrogen column density $\mathrm{N}_{H}=1.5 \times 10^{25} \mathrm{~cm}^{-2}$ to fit the SIGMA/ART-P observation results. 


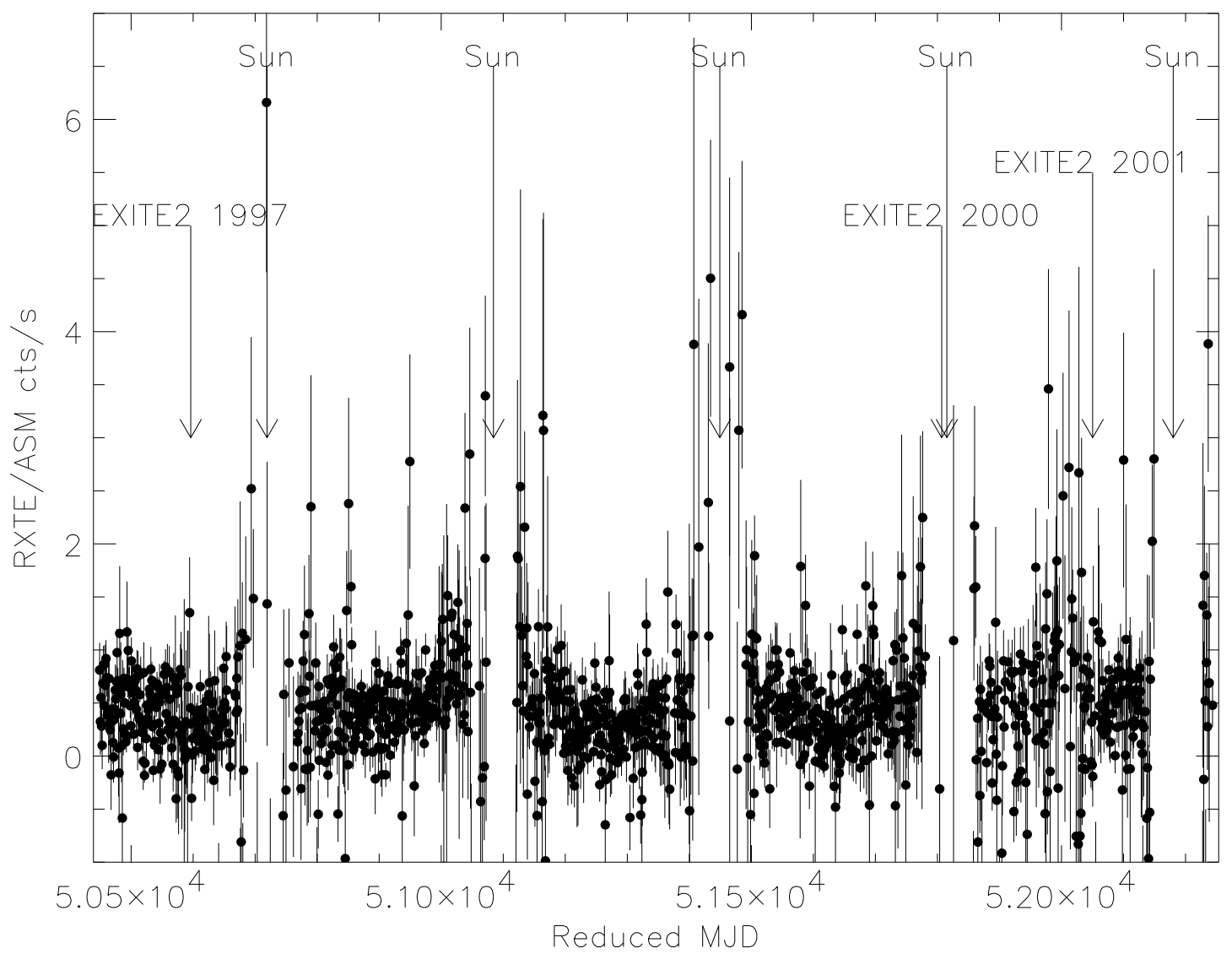

Fig. 4.- RXTE/ASM light curve for 3C273 showing times of EXITE2 observations of the field and 1997 detection vs. 2000 and 2001 upper limits of GRS1227+025. The increased ASM count rate due to solar background is evident for the times (marked) when the Sun is closest $\left(\sim 4^{\circ}\right)$ to $3 \mathrm{C} 273$ or GRS1227+025. 